\title{
NOTAS E INFORMACIÓN
}

\section{Nota crítica a dos lugares oscuros de Ovidio (Met. XIII 884 y 890 )*}

A critical note on two problematic passages of Ovid (Met. XIII 884 and 890)

\section{Luis Rivero García}

Universidad de Huelva

lrivero@uhu.es

ORCID iD: http://orcid.org/0000-0001-7140-2072

Se analizan dos pasajes de las Metamorfosis de Ovidio y se sugieren propuestas de enmienda.

Palabras clave: Ovidio; Metamorfosis; crítica textual.

Two passages of Ovid's Metamorphoses are considered, and an attempt is made to correct them.

Key words: Ovid; Metamorphoses; textual criticism.

Cómo citar este artículo / Citation: Rivero García, Luis 2017: «Nota crítica a dos lugares oscuros de Ovidio (Met. XIII 884 y 890)», Emerita 85 (1), pp. 153-159.

Ou., Met. XIII 882-892:

Insequitur Cyclops partemque e monte reuulsam

mittit, et extremus quamuis peruenit ad illum

angulus †e saxo†, totum tamen obruit Acin.

At nos - quod fieri solum per fata licebat -

* El presente artículo, elaborado en los primeros meses de 2015 en la Academia Americana de Roma y en la Biblioteca Vaticana gracias a una beca del Ministerio de Educación, se inserta en el Proyecto de Investigación FFI2013-42529, financiado por el MICINN, Gobierno de España. El texto y aparato crítico, así como la propia argumentación pertenecen al comentario crítico textual que actualmente preparo sobre el libro XIII de las Metamorfosis. Los manuscritos, agrupados por bloques cronológicos, así como las ediciones se citan de forma abreviada, siguiendo las propuestas disponibles en http://www.uhu.es/proyectovidio/ esp/index.html. El texto de Planudes se cita por la edición de Papathomopoulos-Tsavare 2002. 
fecimus ut uires adsumeret Acis auitas.

Puniceus de mole cruor manabat, et intra temporis exiguum rubor euanescere coepit fitque color primo turbati fluminis imbre purgaturque mora. Tum moles iacta dehiscit uiuaque per rimas proceraque surgit harundo osque cauum saxi sonat exsultantibus undis

- $\underline{84}$ e saxo $\Omega$, edd.; uix sanum : ex saxo V2 B4Ba (p.c.)CDeDrFGgO3V5V9(p.c. a m.p.) Cs2Li3P8 O10²(i.l. p.c.) P41, prob. Ellis, Edwards 1905 : is motus M(p.c., -us i.ras.; quid a.c. n.l.) Lr27 : asaxo AbMoV9(a.c.) Ft : a saxo Bs4 Es4 : exaxo B3 : exasto $B a($ a.c.) : est saxo Lr8 O10 Es6 : quid $N$ a.c. (e saxo i.ras.) n.l. : is montis Merkel 1850 : is molis Merkel 1875, Zingerle 1884, Polle 1888, H-K-E 1898, Keene 1898, Magnus 1914, Ehwald 1915, van Proosdij 1951, H-K-E-A 1966, Fink 2007 : hic iactu Riese 1872(is i. dub. in notis) : ex iactu Riese 1889 : exiguus Hellmuth - $\underline{890}$ iacta B2Ld Sp Rd, Plan., malit Glareanus, prob. Heinsius 1659(qui et «in ... tribus» test.), Burm. 1727, Walch. 1731, Jahn 1832, Weise 1845, Koch 1866, H-K-E 1898, Goold 1984 : tacta $\Omega$, Puteol. 1471, Venet. 1472, Calph. 1474, Accurs. 1475, Regius 1493, Aldina 1502, Naug. 1516, Ciof. 1575, Heinsius 1652, Magnus 1914, Ehwald 1915(iacta H-K-E 1898), Lafaye 1930, Trepat-de Saav. 1932, van Proosdij 1951, Breitenbach 1964, H-K-E-A 1966, Anderson 1982, Segura 1983, Hill 2000, Hopkinson 2000, R. de Verger 2005, Tarrant 2004(qui tamen suspectum hab.), dub. Lemaire 1822(in notis) : tales Li3 : fracta $A L u A 4^{3}$ (mg.)Ba(p.c.)DeFe (mg. u.l.) $G^{3}(m g .$, add. «i. aperta»)L4(tacta a.c.)LsN2TrVd(p.c., fort. a m.p.) B8Ds2 (i.l. u.l.) P10So BoBo2Es22(p.c.)P28(p.c.) B14Es5 Mv7, "prim. Moret. et quindecim alii» test. Heinsius 1659, prob. Gierig 1807, Bothe 18182, Lemaire 1822, Richter 1828, Baumg.-Crus. 1834, Bach 1836, Loers 1843, Merkel 1850, Riese 1872, Korn 1880, Zingerle 1884, Polle 1888, Simmons 1889, Edwards 1905, Fabbri 1923, Fink 2007, dub. Tarrant 2004(in app.) : facta $G^{2}$ (p.c.; tacta G) Ba(a.c.) : fata $E s^{3}:$ tracta FO3(per comp.)V4²(r suprascr.)V5(tacta p.c.) O10(tacta p.c.)P28(a.c.), «in utroque meo, Urs. Farnes. et cod. Vatican.» test. Ciof. 1575, dub. Ellis : tecta Lr3(tacta p.c.) Lr22V30, Aler. 1471, Lugd. 1546, Lugd. 1565, Glar-Long. 1570, Bersm. 1596 : tanta A3 To(tacta $m g$. u.l. $\left.T o^{2}\right)$ : tota $A b\left(\operatorname{tacta}\right.$ i.l. u.l. $\left.A b^{2}\right)$, coni. Hardie : acta Lr4Lr6(uid.) : capta $V d($ a.c.) : rupta $P 41$ : cuncta $B s 4$ (tracta uel tincta i.l. u.l.) : certa «in nonnullis» test. Heinsius 1659 : taetra Merkel 1875 : torta fort. legendum •

En el v. 884, perteneciente al episodio de la muerte de Acis a cargo de Polifemo, el ms. $M$ (s. $\left.\mathrm{XI}^{2}\right)$ nos deja la extraña lectura is motus ( $M^{2}$ sobreescribe «s. angulus»), en la que $-u s$ está escrito sobre algo borrado y hoy ilegible; $V 2\left(\mathrm{~s} . \mathrm{XI}^{2}\right)$ y un grupo de recentiores dan ex saxo, grafía que recibió la aprobación de R. Ellis al hallarla sobreescrita como corrección de est saxo en $\mathrm{O}^{2} 0^{2}$ (s. XIV ${ }^{\mathrm{ex}}$ ), sobre el muy discutible argumento de que «est, proves ex not $e$ to be right» (ap. Simmons 1889, p. 167), y 
esta variante fue llevada a su texto por Edwards (1905, p. 479; para ex saxo cf. e.g. Vitr., VIII 6.8, pero para e saxo cf. e.g. Cato, Orig. II 16); en $N$ (s. XI ${ }^{\text {ex }}-\mathrm{XII}{ }^{\mathrm{in}}$ ) la lectura e saxo está escrita sobre algo borrado que tal vez podría haber sido is motus pero que hoy resulta imposible de determinar con una mínima fiabilidad.

En su $1^{\mathrm{a}}$ edición (1850) Merkel subió al texto su conjetura is montis (cf. 882), no secundada por ningún otro editor, pero en la $2^{\mathrm{a}}$ editó is molis a partir de $887 \mathrm{y}$ 890 (cf. VIII 357: véase Merkel 1875, p. XXXVIII), retrotrayendo la coma a illum (v. 883), conjetura que sí ha sido seguida por Ehwald, Magnus y otros editores hasta nuestros días. Riese, por su parte, editó hic iactu en su $1^{\mathrm{a}}$ edición, aunque dejando abierta en sus notas la posibilidad de leer alternativamente is iactu (1872, p. XXIV). En su $2^{a}$ edición cambió de idea y sostuvo $(1889$, p. XXVI) que la parádosis e saxo era corrupción de ex iactu a través de una grafía facxo. Por último, Hellmuth (1880, pp. 28-29) comienza señalando lo inaceptable de la expresión angulus e saxo en lugar de angulus saxi y a continuación aborda las limitaciones de las propuestas de Merkel y de Riese, sobre todo en lo relativo a los pronombres mostrativos y fóricos, para a continuación proponer la interesante conjetura angulus exiguus, estilísticamente lograda por la yuxtaposición significativa exiguus totum, que él apoya en paralelos como VIII 337: longa paruae, XI 506: inferno summum, o XII 495: in hunc omnes unum. Además, el adjetivo podría evocar el modelo virgiliano (Aen. X 128): haud partem exiguam montis. Por lo que respecta a la doble adjetivación de angulus, Hellmuth recuerda que ésta no es obstáculo cuando uno de los adjetivos tiene valor local, para lo que compara XI 197-198: dextera Sigei, Rhoetei laeua profundi / ara Panomphaeo uetus est sacrata Tonanti, y XV 443: externum patria contingat amicius aruum. Desde el punto de vista paleográfico la conjetura es creíble respecto de e saxo, aunque Hellmuth, tratando de dar cuenta de la lectura de $M$, explica la corrupción a partir de una repetición de extremus, del verso anterior, lo que habría forzado a los copistas a rehacer el texto, siendo el copista de $M$ el más «audaz» en su reconstrucción, pero esto no deja de ser una forma de reconocer que no se sabe cómo pudo escribir aquel copista lo que escribió. La propuesta de Hellmuth, más fina que las de Merkel y Riese, tiene sin embargo el inconveniente de ser redundante, pues la expresión extremus angulus tiene ya la precisa finalidad de indicar que lo que cayó sobre Acis fue una parte exigua - un borde, un pico- de la porción de monte arrancada por Polifemo.

Aun así, sigue siendo evidente que e saxo no va bien porque, como acertadamente señalaba Hellmuth, aquí tendría que equivaler a un simple genitivo («ungewöhnliche adnominale partitive e», según Bömer 1982, p. 448, sin más argumentación), y eso no es aceptable en el latín de Ovidio (y tampoco tiene sentido como separativo ni dependiendo de peruenit ni de angulus), pero además porque e saxo es una expresión rara y limitada a la prosa con la sola excepción de este pasaje y porque aparece sospechosamente cerca de e monte (v. 882). 
El sintagma e saxo podría ser deformación gráfica de la lectura genuina, pero también podría proceder de una glosa a un participio concertado con angulus del tipo excisus, exceptus, excussus o exsectus, i. e. «una punta marginal desgajada (de la roca)». Del mismo modo, is motus podría ser glosa de un participio, pero en este caso concertado con el sujeto de obruit, como eiectus (aunque con el sentido de 'arrojar violentamente' Ovidio prefiere eiectare: cf. II 231, V 353), exactus (véase Bömer 1976, pp. 214 y 265, coment. a IV 734 y V 171), excissus (aquí con el sentido de 'arrojado': cf. e.g. Tac., Hist. IV 23.3), exceptus (aquí, 'recibido') o inmissus.

La lectura de $M$ (y tal vez -recuérdese- de $N$ a.c.) también podría ser deformación gráfica o bien glosa de un participio del tipo euoluens (cf. XII 519), inuolitans, intortus, o de una locución como in gyrum/-os, i.e. «aunque llega hasta él el extremo del borde girando» (cf. e.g. Verg., Aen. VII 378-381), que habría podido venir sugerido a Ovidio por é $\pi \imath \delta \imath v \dot{\sigma} \sigma \alpha \varsigma$ ('haciéndola girar': véase Heubeck 1992, p. 41) en el

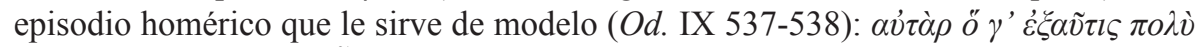

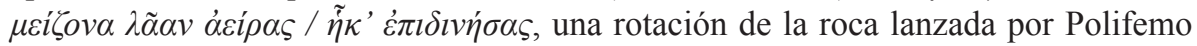
que se corresponde al conocido movimiento de taladro con el que Odiseo y los suyos le habían hundido la estaca en el ojo (Od. IX 383-386, con Heubeck 1992, p. 34, coment. a 382-390; E., Cyc. 460-463; Verg., Aen. III 635). No encuentro, sin embargo, ningún pasaje dentro o fuera de la obra de Ovidio que avale con suficiente nitidez ninguna de estas propuestas, por lo que de momento me limito a señalar como corrupto el texto transmitido.

El segundo pasaje objeto de discusión está en el v. 890 y puede tener, como veremos, alguna relación con el anterior. La lectura ampliamente mayoritaria de los manuscritos es moles tacta, que también ha sido adoptada por una mayoría de editores (uid. app.), tanto entre las ediciones más antiguas como por parte de Heinsius en su $1^{\mathrm{a}}$ edición (1652), y muy especialmente desde que Magnus la recuperara con el discutible argumento de que se trata de un «toque mágico» $(1914$, p. 524: «a me sc, cf 652, 886, XI 308»; véase además Bömer 1982, p. 449), lo que en todo caso habría necesitado de un agente más explícito (véase Hill 2000, p. 168; Hopkinson 2000, p. 230; Hardie 2015, p. 358; todos con dudas respecto de la lectura). El último editor que la ha mantenido ha sido Tarrant, quien sin embargo muestra (2004, p. 406 in app.) sus dudas sobre ella y sus simpatías por fracta («fort. recte»; una posición inversa vemos en Lemaire 1822). Copistas y editores han sentido la necesidad de glosar el participio, lo que es indicio de que no se encuentran del todo cómodos con él. Así, por ejemplo, en $N^{2}$ encontramos sobreescrito unda, en $M 2^{2}$ vemos a sanguine (sanguine en $\mathrm{V}^{2}$ ) y en $\mathrm{Fe}^{2}$ leemos ab aqua. Regius, por su parte, no especifica el origen del contacto (1493, ad loc.): «Tum rupes percussa aperitur».

Otra de las variantes mejor atestiguadas es fracta, que no fue llevada a su texto pero sí recibió el beneplácito («bene») de Heinsius (1659, pp. 354-355) y que fue editada por Gierig $(1807$, p. 303$)$ por comparación con XII 488: fractaque dissiluit 
percusso lammina callo, y con Verg., G. IV 410: in aquas tenues dilapsus abibit, pasajes ambos de muy escaso valor probatorio (cf. Luck 2009, p. 113). Esta lectura ha sido aceptada por algunos editores hasta nuestros días. Bach $(1836$, p. 362) cree que de ella surgió tracta y de ésta a su vez tacta. El problema es que fracta dehiscit es una redundancia que delata el origen espurio de fracta (cf. rupta en el ms. P41).

Otra variante interesante, la de grafía más cercana a tacta, es iacta, que he encontrado tan sólo en $B 2 L d S p R d$ y que debió de encontrar Planudes en su antígrafo, pues tradujo $\beta \lambda \eta \theta \varepsilon \tilde{\varepsilon} \sigma \alpha$. Esta lectura recibió la aprobación de Glareanus (Glar.-Long. 1570, p. 228: «Omnes tacta legunt. At ego iacta malim. Intelligo enim saxum a Polyphemo iactum»; cf. Bersm. 1596, p. 547) y fue llevada a su texto por Heinsius en su $2^{\text {a }}$ edición a partir de la paráfrasis «iactis in altum molibus» $(1659$, p. 355), paráfrasis desde luego innecesaria frente a la más simple de Glareanus. Entre los editores recientes tan sólo Goold (1984; así ya Miller 1921²) la ha aceptado.

Mucho menos creíble resulta la variante tracta, pues la piedra fue arrojada, como se dice en el v. 883 (mittit), y no arrastrada (compárese además la variante acta). Ciofanus $(1575$, p. 187) la atestigua en varios de sus manuscritos y esta variante recibió la tímida aprobación de Ellis, quien la había encontrado de nuevo en 010 (ap. Simmons 1889 , p. 168). Otras variantes transmitidas son tecta, tanta, facta, capta, tincta, cuncta o certa. Semánticamente cercana a tanta y, más aún, a cuncta está la variante tota, que he encontrado en $A 3$ y en To y que Tarrant (2004, p. 406) recogía como propuesta de Hardie (el propio Hardie 2015, p. 72 la recoge in app., aunque no la lleva al texto ni la menciona en su comentario: p. 358). Si paleográficamente es viable, tiene además a su favor la yuxtaposición significativa tota dehiscit. Muy desafortunada, en cambio, fue la propuesta taetra de Merkel para su $2^{\mathrm{a}}$ edición (1875; en 1850 había editado fracta).

La parádosis tacta tal vez nos permitiría sugerir torta (i. e. torta $>$ tarta $>$ tacta), pues la roca fue arrojada (cf. IV 709-710: quantum Balearica torto / funda potest plumbo medii transmittere caeli; epist. IV 158: ueniant proaui fulmina torta manu), quizá incluso con efecto giratorio, tal como apuntábamos más arriba en nuestra discusión sobre e saxo. Ante las limitaciones de tacta me inclino entre dudas por iacta como variante más probable tanto por el significado como por la grafía, que habría dado lugar a tacta y a las demás a partir de ésta.

\section{BiBLIOGRAFÍA}

Bach, E. C. Chr. 1836: P. Ovidii Nasonis Metamorphoseon libri XV, mit kritischen und erläuternden Anmerkungen von ... Vol. II, Hannover.

Bersman, G. 1596: Publ. Ovidii Nasonis Operum Tomus II. quo continentur Metamorphoseon libri XV. Ex postrema Iacobi Micylli recognitione, et recensione nova Gregorii Bersmani ..., editio tertia aliquot locis auctior, Leipzig. 
Bömer, F. 1976: P. Ovidius Naso. Metamorphosen. Kommentar von ... Buch IV-V, Heidelberg. Bömer, F. 1982: P. Ovidius Naso. Metamorphosen. Kommentar von ... Buch XII-XIII, Heidelberg.

Ciofanus, H. 1575: Herculis Ciofani, Sulmonensis, in P. Ovidii Metamorphoseon ex XVII antiquis libris Observationes ..., Venecia.

Edwards, G. M. 1905: Corpus poetarum Latinorum a se aliisque denuo recognitorum et brevi lectionum varietate instructorum edidit I. P. Postgate. Tom. I. Editio altera emendatior (= 1894). Metamorphoseon libri XV, recogniti a G. M. Edwards, Londres.

Ehwald, R. 1915: P. Ovidius Naso. Opera. Vol. II, Metamorphoses, editio maior, Leipzig.

Gierig, G. E. 1807: P. Ovidii Nasonis Metamorphoses. Recensuit varietate lectionis notisque instruxit et indices duos, unum verborum, alterum nominum propriorum adiecit Gottlieb Erdmann Gierig. Editio altera ad singulos prope versus vel emendatior vel auctior. Tomus posterior [Met. IX-XV], Leipzig.

Glareanus, H. 1570: P. Ovidii Nasonis opera, veterum exemplarium auxilio ab infinitis mendis emendata. Henrici Glareani annotationes in Metamorphosin \& ad verba \& ad res intelligendas magni usus. Praeterea Longolij, quae lectorem plurimum in impeditis locis iuuare possunt. Item, fragmenta quaedam Ouidij ex libris, qui magna parte periere, epigrammaton $\&$ non male natum carmen ad Pisonem. Excudebat Ioannes Kyngstonus (s.l.).

Goold, G. P. 1984: Ovidius. Metamorphoses. With an English Translation by F. J. Miller, Revised by G. P. Goold, Londres - Cambridge (Mass.).

Hardie, Ph. 2015: Ovidio, Metamorfosi, volume VI (libri XIII-XV), a cura di Ph. Hardie, testo critico basato sull'ed. oxoniense di R. Tarrant, trad. di G. Chiarini, Milán.

Heinsius, N. 1652: Operum P. Ovidii Nasonis editio nova, accurante Nicolao Heinsio, Dan. fil., II, Amsterdam.

Heinsius, N. 1659: Operum P. Ovidii Nasonis editio nova. Nic. Heinsius, Dan. F. recensuit, et notas addidit, II, Amsterdam.

Hellmuth, C. 1880: Emendationsversuche zu Ovids Metamorphosen, Progr. Caesareolutreanum.

Heubeck, A. 1992: A Commentary on Homer's Odyssey, vol. II (books IX-XVI), Oxford.

Hill, D. E. 2000: Ovid. Metamorphoses XIII-XV and indexes, ed. with an introd., transl. and notes by ..., Warminster.

Hopkinson, N. 2000: Ovid: Metamorphoses. Book 13, ed. by ..., Cambridge - Nueva York.

Lemaire, N. E. 1822: Publius Ovidius Naso, ex recensione Gott. Erdmann Gierig cum variis lectionibus codicum Parisinorum cui novas addidit notas Nicol. Elig. Lemaire; vol. IV: Met. 8-15, París.

Luck, G. 2009: «More missing letters in Ovid's Metamorphoses», MH 66, pp. 88-119.

Magnus, H. 1914: P. Ovidi Nasonis Metamorphoseon libri XV, Berlín.

Merkel, R. 1850: P. Ovidius Naso, ex recognitione R. Merkelii, vol. II, Leipzig.

Merkel, R. 1875: P. Ovidius Naso, ex iterata R. Merkelii recognitione, vol. II, Leipzig.

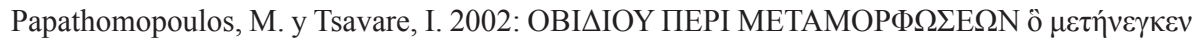

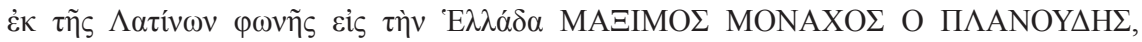
Atenas.

Regius, R. 1493: P. Ouidii Metamorphosis, ed. Raph. Regius, Venecia.

Riese, A. 1872: P. Ovidii Nasonis Carmina, edidit ... vol. II. Editio stereotypa, Leipzig. 
Riese, A. 1889: P. Ovidii Nasonis Carmina, edidit ... vol. II. Editio stereotypa iterum recognita, Leipzig.

Simmons, Ch. 1889: P. Ovidii Nasonis Metamorphoseon XIII. XIV, edited with Introduction, Analysis and Notes by ..., Londres y Nueva York.

Tarrant, R. J. 2004: P. Ouidi Nasonis Metamorphoses, Oxford.

Fecha de recepción de la primera version del artículo: 07/01/2016

Fecha de aceptación: 25/01/2016

Fecha de recepción de la version definitiva: 10/02/2016 